\title{
Oxidative Stress in Cardiovascular Pathologies: Genetics, Cellular, and Molecular Mechanisms and Future Antioxidant Therapies
}

\author{
Adrian Manea, ${ }^{1}$ Ana Fortuno, ${ }^{2}$ and Jose Luis Martin-Ventura ${ }^{3}$ \\ ${ }^{1}$ Department of Genomics, Transcriptomics, and Molecular Therapies, Molecular and Cellular Pharmacology, Functional Genomics \\ Laboratory, Institute of Cellular Biology and Pathology "Nicolae Simionescu," 8 BP Hasdeu Street, 050568 Bucharest, Romania \\ ${ }^{2}$ Division of Cardiovascular Sciences, Center for Applied Medical Research, University of Navarra, Pio XII, \\ 31008 Pamplona, Spain \\ ${ }^{3}$ Vascular Research Laboratory, Fundación Jiménez Diaz, Autonomous University of Madrid, Avenida Reyes Católicos 2, \\ 28040 Madrid, Spain
}

Correspondence should be addressed to Adrian Manea, adrian.manea@icbp.ro

Received 23 December 2012; Accepted 23 December 2012

Copyright (C) 2012 Adrian Manea et al. This is an open access article distributed under the Creative Commons Attribution License, which permits unrestricted use, distribution, and reproduction in any medium, provided the original work is properly cited.

Reactive oxygen species (ROS) are generated during normal cell physiology; however increased ROS formations are highly detrimental in many cardiometabolic disorders, including atherosclerosis, hypertension, diabetes, obesity, neurodegenerative diseases, and aneurism [1-3]. Despite the numerous existing data the role of ROS and the associated redox mechanisms of the disease inception and progression remain elusive. A common feature of all cardiovascular disorders is the occurrence of oxidative stress, a condition characterized by an imbalance between ROS production and the ability of biological systems to detoxify the reactive intermediates [4]. Produced in excess, ROS react indiscriminately and cause irreversible damage of the majority of biological molecules, thereby altering cell functions [5]. Although extensive studies have focused on the redox control of vascular response to inflammatory and metabolic insults, the molecular mechanisms ROS generation and the way that this class of molecules contributes to vascular damage are not entirely clear. Therefore, uncovering the intimate molecular control mechanisms involved in regulation of the delicate balance of ROS formation and neutralization in the vascular wall cells is a prerequisite for the development of an effective antioxidative stress therapy.

The topic of this special issue focuses on recent advances in experimental aspects of oxidative stress in cardiovascular disorders. In the current issue, $\mathrm{H}$. Osorio et al. have addressed the role of sodium-glucose cotransporter (SGLT2) in the oxidative and nitrative stress processes taking place in the kidney of diabetic rats. The authors have shown that
SGLT2 inhibition reverse streptozotocin-induced diabetes and redox disbalance in the kidney, suggesting the potential therapeutic role of SGLT2 inhibitors in diabetic nephropathy. The role of oxygen supplementation in systemic redox balance in rats has been investigated in the paper by $\mathrm{F}$. Nagatomo et al. The authors demonstrated that exposure to oxygen concentrations higher than $40 \%$ leads to a prooxidant response able to produce morphological changes in red blood cells, whereas antioxidant response does not seem to be modified in the $24 \mathrm{~h}$ period of the study. J. Dudka et al. have evaluated the implication of hypothyreosis on the oxidative stress induced by doxorubicin. The authors also analyzed the effect of both hypothyreosis and the drug on cytochrome P450 NADPH-reductase and inducible nitric oxide synthase expression. The results of this study bring additional support that hipothyroid conditions may increase the cardiac oxidative stress caused by doxorubicin. In the paper by M. Al-Shebly and M. Mansour the authors have analyzed whether oxidative stress status is modified in hypertensive/diabetic women during labor. The findings of this study clearly demonstrate that different biomarkers of redox balance-altered in hypertensive/diabetic women during labor and suggest that this redox imbalance could increase the risk of fetal abnormalities. In the paper of S. Muhammad et al., the authors aimed at investigating the antioxidant effects of mineral elements supplementation using a rat model of hypertension. They showed that copper, manganese, and zinc supplementation reduces the blood pressure as compared with hypertensive control. 
In addition, a significant reduction in the plasma total cholesterol, triglyceride, low density lipoprotein-cholesterol, very low density lipoprotein-cholesterol, malondialdehyde, and insulin level, as well as increases in the high density lipoprotein cholesterol, total antioxidant activities, and nitric oxide have been demonstrated in the supplemented groups relative to the hypertensive control. Together, the present report highlights that various minerals may play a role in preventing oxidative stress, dyslipidemia, and insulin resistance associated with hypertension. Interestingly, R. Wu et al. demonstrated the beneficial effects of chronic acetylsalicylic acid (ASA) treatment on cardiac hypertrophy and oxidative stress in cardiomyopathic hamsters. The authors concluded that ASA present a therapeutic potential to prevent cardiac dysfunction.

The review of J. Madrigal-Matute et al. is focused on the thioredoxin (TRX) system, which is composed of several proteins such as TRX and Peroxiredoxin (PRDX). In addition to their main role as antioxidants, recent data highlights their function in several processes involved on the development of atherothrombosis. In fact, since TRX and PRDX are present in the atherosclerotic plaque and can be secreted under prooxidative conditions to the circulation, their role as diagnostic, prognostic, and therapeutic biomarkers of cardiovascular diseases (CVD) has been addressed. In conclusion, J. Madrigal-Matute et al. summarized several findings that demonstrate the major role of the TRX system in the maintenance of the redox status in CVD. Furthermore, the extracellular levels of PRDX/TRX seem to be related with a pro-oxidative scenario suggesting their potential role as biomarkers for oxidative related diseases. Nevertheless, their value as useful therapeutic tools is being tested and future studies are necessary to validate its prospective beneficial effects in CVD.

Vascular NADPH oxidases (Nox) are a class of heterooligomeric enzymes, whose unique function is the generation of ROS in a highly regulated manner [6]; conversely, the specific role of each enzyme is yet to be discovered. Nox expression and activity are significantly upregulated in the vasculature of hypertensive subjects and are associated with the development of macro- and microvascular diseases [7]. Since the members of the Nox family are major triggers of oxidative stress they have a prominent role in the pathology of diabetes-induced vasculopathies $[8,9]$; thus, Nox and their upstream regulators may become important therapeutic targets [10]. In the paper of J. Miguel-Carrasco et al., the authors investigated the effects of the profibrotic factor TGFbeta 1 in mediating Nox overactivity and oxidative stress in hypertensive rats. The results showed that pharmacological inhibition of TGF-beta 1 pathway greatly reduces the Nox activity, expression of the Nox 2 and Nox4 isoforms, and nitrotyrosine levels in hypertensive rats compared to control animals. Collectively, the data of this study provides conclusive evidence on the involvement of this enzyme in the profibrotic actions of TGF-beta 1.

The major cause for diastolic heart failure is cardiac aging, which is referred to a dramatic decline in cardiac pump function with advanced age and resulted in diastolic dysfunction. Current clinical treatment for patients with diastolic heart failure is disappointing. The cardioprotective effects of EGB761, a standard extract from the leaves of Ginkgo biloba, have been demonstrated already. The aim of the present study was to investigate the potential antiaging-associated cellular diastolic dysfunction effects of EGB761, exploring underlying molecular mechanisms. Cardiomyocyte aging model was established by treating with D-galactose. Treatment with EGB761 attenuated the intracellular formation of AGEs, delays the cellular senescence, and increase reuptake of $\mathrm{Ca}^{2+}$ stores in the sarcoplasmic reticulum. J. Liu et al. have suggested that EGB761 may protect against aging-associated diastolic dysfunction in cardiomyocytes. Furthermore, it was possible that EGB761 could upregulate SERCA2a function through improvement of the amount of Ser16 sites PLN phosphorylation. In conclusion, EGB761 significantly improved the diastolic function in cardiomyocytes, through the regulation of myocardial sarcoplasmic reticulum calcium transport regulatory.

NF-E2-related factor 2 (Nrf2) represents a promising therapeutic target to prevent oxidative stress and oxidative damage in various pathologies, including cardiovascular diseases. In the study of E. Donovan et al., the beneficial effects of phytochemicals included in dietary supplements might be an effective strategy to protect the cells against the detrimental effects of oxidative stress. The authors have demonstrated the Protandim activates Nrf2, a condition that ultimately influenced the protection of human coronary artery endothelial cells against an oxidative challenge.

In the paper by A. Ashour et al., the authors have addressed the potential use of metformin to prevent cardiotoxic effects of doxorubicin, a potent antitumor agent, in an experimental model of cardiomyopathy in rats. The adverse effects of doxorubicin include biochemical and morphological cardiac markers of injury, associated to decreasing antioxidant systems. Interestingly, the authors demonstrated that treatment with metformin prevents deleterious changes associated to doxorubicin mainly by modulation of redox balance.

Collectively, the original articles published in this special issue stimulate the ongoing efforts to identify the basic molecular mechanisms regulating the oxidative stress that may be used to find ways to manage its occurrence and correct its adverse effects.

\section{Acknowledgments}

We thank all the authors, the referees, and the staff of Hindawi's Editorial Office for their remarkable contribution that made this special issue possible.

\section{Adrian Manea \\ Ana Fortuno \\ Jose Luis Martin-Ventura}

\section{References}

[1] D. D. Heistad, Y. Wakisaka, J. Miller, Y. Chu, and R. Pena-Silva, "Novel aspects of oxidative stress in cardiovascular diseases," Circulation Journal, vol. 73, no. 2, pp. 201-207, 2009. 
[2] M. Simionescu, "Implications of early structural-functional changes in the endothelium for vascular disease," Arteriosclerosis, Thrombosis, and Vascular Biology, vol. 27, no. 2, pp. 266274, 2007.

[3] J. Madrigal-Matute, C. E. Fernandez-Garcia, C. GomezGuerrero et al., "HSP90 inhibition by 17-DMAG attenuates oxidative stress in experimental atherosclerosis," Cardiovascular Resarch, vol. 95, no. 1, pp. 116-123, 2012.

[4] A. Fortuño, J. Bidegain, A. Baltanás et al., "Is leptin involved in phagocytic NADPH oxidase overactivity in obesity? Potential clinical implications," Journal of Hypertension, vol. 28, no. 9, pp. 1944-1950, 2010.

[5] A. Manea and M. Simionescu, "Nox enzymes and oxidative stress in atherosclerosis," Frontiers in Bioscience, vol. 4, pp. 651-670, 2012.

[6] A. Manea, "NADPH oxidase-derived reactive oxygen species: involvement in vascular physiology and pathology," Cell and Tissue Research, vol. 342, no. 3, pp. 325-339, 2010.

[7] A. C. Montezano and R. M. Touyz, "Molecular mechanisms of hypertension-reactive oxygen species and antioxidants: a basic science update for the clinician," Canadian Journal of Cardiology, vol. 28, no. 3, pp. 288-295, 2012.

[8] U. Förstermann, "Oxidative stress in vascular disease: causes, defense mechanisms and potential therapies," Nature Clinical Practice Cardiovascular Medicine, vol. 5, no. 6, pp. 338-349, 2008.

[9] I. M. Fearon and S. P. Faux, "Oxidative stress and cardiovascular disease: novel tools give (free) radical insight," Journal of Molecular and Cellular Cardiology, vol. 47, no. 3, pp. 372-381, 2009.

[10] I. M. Fenyo, I. C. Florea, M. Raicu, and A. Manea, "Tyrphostin AG490 reduces NAPDH oxidase activity and expression in the aorta of hypercholesterolemic apolipoprotein E-deficient mice," Vascular Pharmacology, vol. 54, no. 3-6, pp. 100-106, 2011. 


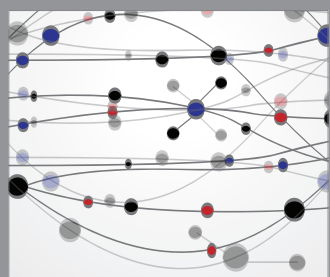

The Scientific World Journal
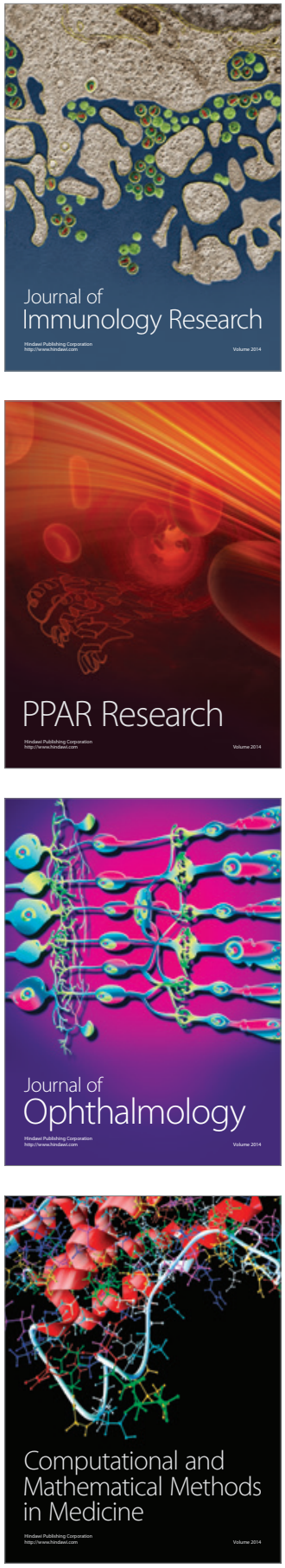

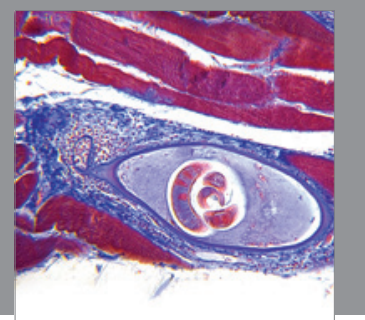

Gastroenterology

Research and Practice
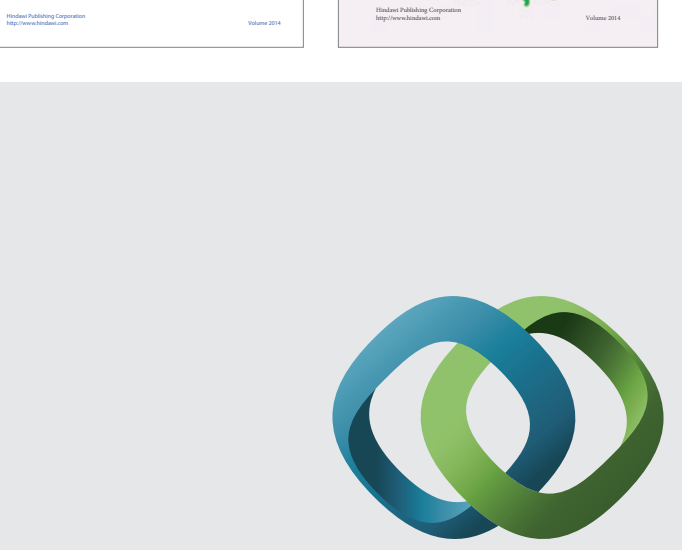

\section{Hindawi}

Submit your manuscripts at

http://www.hindawi.com
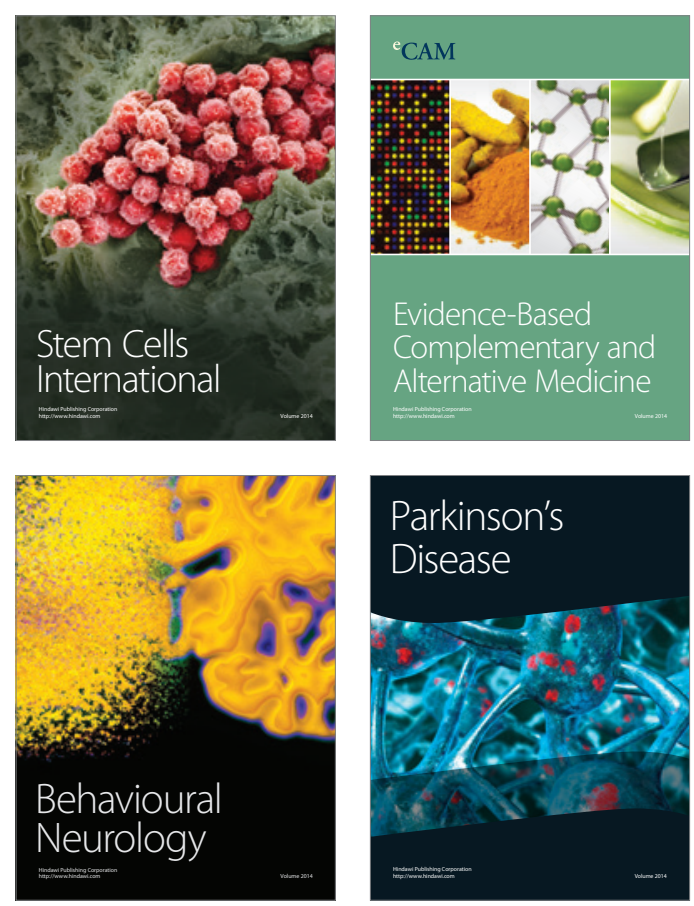

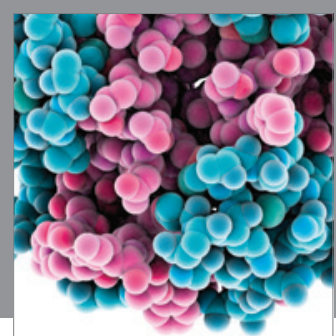

Journal of
Diabetes Research

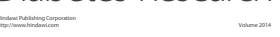

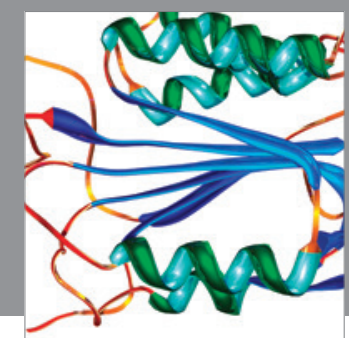

Disease Markers
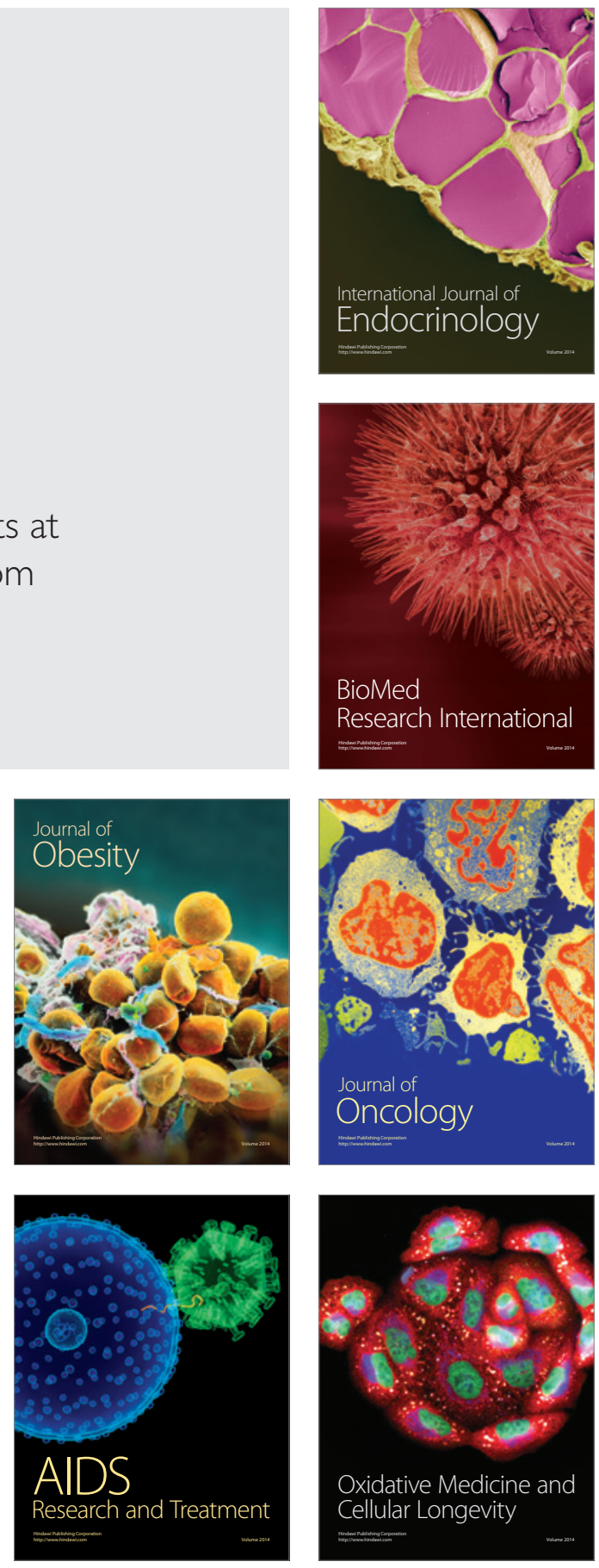